\title{
The impact of asthma exacerbations on health-related quality of life in moderate to severe asthma patients in the UK
}

\author{
Andrew Lloyd ${ }^{\mathrm{a}, *}$, David Price ${ }^{\mathrm{b}}$, Ruth Brown ${ }^{\mathrm{a}}$
}

a United BioSource Corporation, 20 Bloomsbury Square, London WC1A 2NS, UK

b GPIAG Professor of Primary Care Respiratory Medicine, Department of General Practice and Primary Care, University of Aberdeen, Aberdeen, UK

Received 13 July 2006; accepted 20 November 2006

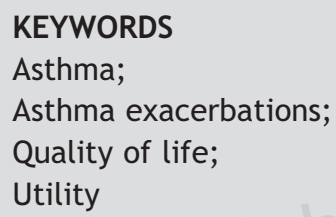

\begin{abstract}
:
Introduction: The objective of this study was to report the impact of exacerbations on health-related quality of life (HRQL) and health utility in patients with moderate to severe asthma (BTS levels $4 \& 5$ ) in the UK.

Materials and Methods: Prospective data regarding HRQL were collected $(n=112)$ using the EQ-5D, mini Asthma Quality of Life Questionnaire (mAQLQ), and Asthma Symptom Utility (ASUI) measures.

Results: The mAQLQ, EQ-5D and ASUI were all significantly worse for patients suffering exacerbations $(p<0.001)$ compared to those without. There was also evidence of a further HRQL decrement in those patients who had been admitted to hospital as a result of an exacerbation during the four-week study.

Conclusions: This study documents the impact of asthma exacerbations on HRQL in patients with moderate to severe asthma. There was some evidence of floor effects on the MAQLQ and ASUI in their ability to capture the impact of exacerbations. These study data are suitable for use in economic evaluations. (c) 2006 General Practice Airways Group. All rights reserved.
\end{abstract}

* Corresponding author. Tel.: +44 (0) 207299 4554; fax.: +44 (0) 2072994555

E-mail address: andrew.lloyd@unitedbiosource.com

\section{Introduction}

Asthma exacerbations are characterised by an increase in asthma symptoms. This can have a significant effect on health-related quality of life (HRQL) and patients' ability to complete usual activities. ${ }^{1,2}$ An asthma exacerbation usually requires an increase in medical therapy, but if more severe, an exacerbation may necessitate hospital admission and can even be fatal. Patients can be classified in terms of current control of 
their asthma symptoms. Control of asthma symptoms and prevention of asthma exacerbations has been shown to be a realistic target for medical treatment albeit not achievable in all patients. ${ }^{3}$

In the UK, the prevalence of asthma and the symptoms of asthma have been shown to be increasing. ${ }^{4}$ Approximately $46 \%$ of asthma patients have intermittent disease (step 1 severity), while the categories 'mild persistent', 'moderate persistent', and 'severe persistent' (GINA steps 2-4) account for $18 \%$ each of the total asthma population. ${ }^{5}$ There were an estimated 71,000 admissions to hospital in 2004 for asthma, and $6 \%$ of individuals with asthma reported an emergency medical visit for their asthma during the previous month. ${ }^{6,7}$

Asthma patients can experience a considerable personal burden in terms of the impact of the disease on HRQL and loss of functioning. There is also evidence that patients who are at risk of exacerbations have significantly worse HRQL compared to other patients. Magid et $a l^{8}$ surveyed 1406 adult asthma patients over 12 months and reported that the 143 who were admitted to the Emergency Department as a result of an exacerbation had significantly worse HRQL scores at the start of the study.

The present study is designed to assess further the HRQL impact of exacerbations in patients with moderate to severe asthma. The study was restricted to more severe patients because we wanted to capture the effects of as many exacerbations as possible. Three measures of HRQL were used, including the mini Asthma Quality of Life Questionnaire (mAQLQ), the Asthma Symptom Utility Index, and the EQ-5D. The EQ-5D is used to derive a societal weighted utility value which can be used to weight survival in the estimation of quality adjusted life years (QALYs) for economic evaluations (referred to here as the EQ- $5 D_{\text {index }}$ ).

The study was purposefully designed as an observational study to capture accurate data regarding the HRQL burden of exacerbations in moderate to severe asthma patients in the UK. In addition, it was designed to recruit 150 patients with step 4 or 5 asthma according to the British Thoracic Society/SIGN Guidelines classification. ${ }^{9}$ These data could be used in economic evaluations of treatments designed to reduce exacerbations.

\section{Materials and methods}

\section{Data collection}

This was a prospective observational study with clinical and HRQL data collected over four weeks.
The short observational period was chosen so that the burden of an exacerbation could be accurately captured as it happened, rather than recalled at some later date. It also reduced the burden for patients in comparison to longer studies and helped to minimise drop-out.

Four asthma centres across the UK were recruited into the study. All patients were recruited through hospital outpatient clinics and primary care offices. To be eligible for the study patients had to have a diagnosis of moderate or severe asthma defined as British Thoracic Society level 4 or $5 .{ }^{9}$ This includes patients who are managed with: at least one high dose formulation of inhaled corticosteroids (ICS) combined with any oral or inhaled long-acting beta agonist (LABA) or any leukotriene-receptor antagonist, or theophyline (Level 4); or, regular oral steroid usage combined with ICS and LABA (Level 5). Efforts were also made to recruit patients with a history of exacerbations, although no patients were currently experiencing an exacerbation when they entered the study. All patients provided written informed consent. Patients were excluded if they were already involved in a therapeutic investigational study, had a history of smoking $\geq 10$ pack years, had active lung disease (e.g., COPD) other than allergic asthma, or had known significant co-morbid conditions such as cancer or depression. Patients with known depression were excluded from the study because of the potential bias effect this could have on the completion of patient-reported outcomes, but no formal psychiatric screening was undertaken.

Data collected prospectively included: any asthma-related hospital admissions; scheduled and unscheduled visits to their general practitioner (GP), respiratory specialist, nurse or hospital casualty department (Emergency room); and prescriptions. These data were all collected in a patient diary. Baseline lgE level was recorded in order to assess patients' atopic status. Patients returned the completed diary cards after four weeks of data collection. Two patients were lost to follow-up.

Participants completed three HRQL measures at the beginning and end of the four-week observational period. The mAQLQ is a disease-specific measure of HRQL. It includes 15 questions which are summarised into four domain scores and a mean score. ${ }^{10}$ The four domains assess HRQL with respect to the impact of environmental factors and activities on asthma, and the burden of asthma in terms of symptoms and patients' emotions. The EQ$5 \mathrm{D}$ questionnaire is a self-administered generic preference weighted HRQL measure..$^{11,12}$ It is summarised as a single-index utility-based score (where $0=$ death and $1=$ perfect health). The EQ-5D also includes a $20 \mathrm{~cm}$ visual analogue scale for 
respondents to rate their own health state (EQ$5 D_{\text {VAS }}$ ). The Asthma Symptom Utility Index (ASUI) is a disease-specific preference-based quality of life measure. ${ }^{13}$ A single index score is derived based upon the patients' preferences for four symptoms (cough, wheeze, shortness of breath, and awakening at night) and two dimensions (frequency and severity). The three HRQL measures used in the current study were summarised according to whether patients experienced an exacerbation and/ or hospitalisation. HRQL data were collected at the study start and at the end of the one-month data collection period. The change in HRQL scores over the one-month period was calculated according to whether patients were hospitalised, had an exacerbation treated with oral steroids, or had no exacerbation.

\section{Analysis}

The patients were segregated in terms of their experience of exacerbations and/or hospitalisations during the study period. An exacerbation was defined as the requirement for at least one course of oral steroids plus an unscheduled visit to a GP, respiratory specialist, or casualty department. In addition, a separate category was included for patients who had been hospitalised due to their asthma during the four weeks of the study.

The HRQL data from the patient sub-groups were compared using one-way analysis of variance (ANOVA) with post-hoc tests (least significant difference, LSD). The sample represents a convenience sample of representative patients from all four centres and was not determined by a formal sample size estimate. Through expert consultation a sample of 100 patients overall was considered sufficiently large to capture a representative sample of moderate to severe patients.

\section{Results}

\section{Participants and demographics}

One hundred and twelve patients completed the HRQL questionnaires at the two time points. General demographics of the group are shown in Table 1. No participant was recorded as having more than one exacerbation during the four-week period. The exacerbations were captured in a four-week window and were defined in terms of oral steroid use and unscheduled visits to the GP or hospital.

\section{HRQL analysis}

Some HRQL data were missing from 23 study participants. Patients were classified into three groups: no exacerbation $(n=85)$; exacerbation without hospitalisation $(\mathrm{n}=22)$; and exacerbations which required hospitalisation $(n=5)$. HRQL data from the last study visit are presented (Table 2). The table also shows the change in HRQL over the one-month course of the study, presented separately for the three groups.

Table 1 includes data on patients' IgE levels which was recorded as an indicator of patients' atopic status. There was no significant difference between the three groups in terms of free IgE level (Kruskal-Wallis test, $\chi^{2}<1$, n.s.). There was also no difference in the proportion of patients with a value over 76 IU IgE $\left(\chi^{2}<1\right.$, n.s. $)$.

The data in Table 2 show the mean scores from the mAQLQ, ASUI and EQ-5D for each group at the end of the four-week period and also the degree of change from baseline. The table is designed to show severity of HRQL impairment for these patients and also the additional impact of experiencing an exacerbation. The data demonstrate that patients

Table 1 Demographics and spirometry data from study participants

\begin{tabular}{lccc}
\hline & No exacerbation & $\begin{array}{c}\text { Exacerbation (no } \\
\text { hospitalisation) }\end{array}$ & $\begin{array}{c}\text { Exacerbation with } \\
\text { hospitalisation }\end{array}$ \\
\hline$n$ & 85 & 22 & 5 \\
Age & $40.5(11.6)$ & $41.4(12.0)$ & $48.4(11.0)$ \\
Gender (proportion female) & $60.7 \%$ & $72.7 \%$ & $60 \%$ \\
Baseline FEV & $2.47(0.75)$ & $2.08(1.03)$ & $1.22(0.48)$ \\
Free IgE (Median) & 121.50 & 186.00 & 114.00 \\
Free IgE $>76$ IU & $63.9 \%$ & $68.4 \%$ & $60 \%$ \\
\hline
\end{tabular}


Table 2 HRQL data for asthma patients at final study visit, distinguished by exacerbations requiring oral steroids or hospitalisation ( $\mathrm{F}$ and $\mathrm{P}$ values from the ANOVA)

\begin{tabular}{|c|c|c|c|c|c|}
\hline & & Mean & $\begin{array}{c}\text { Std } \\
\text { Deviation }\end{array}$ & $\begin{array}{c}\text { Oneway } \mathrm{F} \\
(P)\end{array}$ & $\begin{array}{l}\text { Mean change } \\
\text { from baseline }\end{array}$ \\
\hline \multirow[t]{3}{*}{ mAQLQ symptoms } & No exacerbation & 4.78 & 1.29 & $12.36(<0.001)$ & 0.20 \\
\hline & Exacerbation $\mathrm{w} /$ oral steroids & $3.42^{*}$ & 1.54 & & 0.30 \\
\hline & Hospitalised & 2.60 & 0.85 & & 0.10 \\
\hline \multirow[t]{3}{*}{ mAQLQ environment } & No exacerbation & 4.65 & 1.26 & $11.82(<0.001)$ & 0.06 \\
\hline & Exacerbation $\mathrm{w} /$ oral steroids & $3.37^{*}$ & 1.46 & & -0.07 \\
\hline & Hospitalised & 2.50 & 1.50 & & -0.17 \\
\hline \multirow[t]{3}{*}{ mAQLQ emotion } & No exacerbation & 3.68 & 1.06 & $12.40(<0.001)$ & 0.12 \\
\hline & Exacerbation $\mathrm{w} /$ oral steroids & $2.77^{*}$ & 1.26 & & 0.31 \\
\hline & Hospitalised & $1.44^{*}$ & 0.52 & & -0.06 \\
\hline \multirow[t]{3}{*}{ mAQLQ activity } & No exacerbation & 5.75 & 1.18 & $30.73(<0.001)$ & 0.20 \\
\hline & Exacerbation $\mathrm{w} /$ oral steroids & $3.57^{*}$ & 1.72 & & 0.09 \\
\hline & Hospitalised & 2.56 & 1.07 & & -0.13 \\
\hline \multirow{3}{*}{ mAQLQ total } & No exacerbation & 4.72 & 0.98 & $23.17(<0.001)$ & 0.15 \\
\hline & Exacerbation $\mathrm{w} /$ oral steroids & $3.28^{*}$ & 1.29 & & 0.11 \\
\hline & Hospitalised & 2.28 & 0.86 & $S$ & -0.07 \\
\hline \multirow{3}{*}{$\begin{array}{l}\text { Asthma symptom } \\
\text { utility index }\end{array}$} & No exacerbation & 0.75 & 0.20 & $20.18(<0.001)$ & 0.05 \\
\hline & Exacerbation $\mathrm{w} /$ oral steroids & $0.48^{*}$ & 0.27 & & 0.07 \\
\hline & Hospitalised & 0.31 & 0.22 & & -0.08 \\
\hline \multirow[t]{3}{*}{ EQ5D utility } & No exacerbation & 0.89 & 0.15 & $28.69(<0.001)$ & 0.02 \\
\hline & Exacerbation $\mathrm{w} /$ oral steroids & $0.57^{*}$ & 0.36 & & -0.10 \\
\hline & Hospitalised & $0.33^{*}$ & 0.39 & & $-0.20 \dagger$ \\
\hline \multirow[t]{3}{*}{ EQ5D VAS } & No exacerbation & 76.10 & 15.51 & $15.00(<0.001)$ & 2.81 \\
\hline & Exacerbation $\mathrm{w} /$ oral steroids & $56.43^{*}$ & 21.58 & & -1.29 \\
\hline & Hospitalised & 49.00 & 19.49 & & 1.00 \\
\hline
\end{tabular}

who experienced an exacerbation had significantly worse HRQL at the end of the study. Scores were significantly worse still for those patients who were hospitalised. The post-hoc analyses show that exacerbations were associated with significantly lower scores on all subscales of the mAQLQ, the EQ$5 \mathrm{D}$ and the ASUI. The small group of hospitalised patients were significantly worse than the other exacerbation patients on some of the measures (EQ$5 \mathrm{D}_{\text {index }}$ and $\mathrm{mAQLQ}$ Emotion subscale). The data from the EQ- $5 D_{\text {index }}$ also show that patients' health state utility declined significantly more following the start of the study in the exacerbation patients compared with the other patients. Patients who were hospitalised reported a utility change of -0.20 over the four weeks, and those who had an exacerbation requiring oral steroids (but were not hospitalised) reported a -0.10 change. There were no corresponding significant changes in the mAQLQ and ASUI over the four-week period.

A post-hoc estimation of effect size was undertaken with $\mathrm{EQ}-5 \mathrm{D}_{\text {index }}$ score as the primary endpoint. This revealed a partial $\eta^{2}=0.372$. Dividing patients into those who experienced 
exacerbations explained $37.2 \%$ of the variance in EQ- $5 \mathrm{D}_{\text {index }}$ scores.

\section{Discussion}

This study has demonstrated the significant burden of exacerbations for patients with moderate to severe asthma. Patients who experienced an exacerbation during the course of the study reported significantly worse HRQL, and the short study duration meant that it was possible to capture the HRQL burden of exacerbations as patients experienced them. The mAQLQ scores show a significant deterioration in HRQL for patients who experience an exacerbation compared to those who don't. It is generally recognised that a change of 0.50 is a clinically meaningful shift on the AQLQ and the mAQLQ: the decrement in HRQL on all domains of the mAQLQ exceeded that benchmark for those patients experiencing an exacerbation during the study period. The EQ$5 \mathrm{D}_{\text {index }}$ data indicates that experiencing an exacerbation leads to a decrement in utility of 0.32 . Patients who were hospitalised reported an additional mean decline of 0.24 .

The study demonstrates the burden of exacerbations for patients in terms of impact on HRQL. This burden can be considered separately from the burden related to the day-to-day symptoms of asthma. The impact of exacerbations is related to, but also to an extent distinct from, the impact of day-to-day symptoms. Therefore, the relief of day-to-day symptoms will provide an $\mathrm{HRQL}$ or utility gain in addition to any gain related to reducing exacerbations.

One previous study reports preference values for 100 patients with mild to moderate asthma who were asked to rate the severity of various asthma-related health states. ${ }^{14}$ Participants in the study rated the health states using a visual analogue scale (VAS) anchored at 0 (death) and 100 (perfect health). The health states described mild and severe symptom exacerbations and participants' current health status that day. The data demonstrated patients' relative strength of preference for avoiding severe (mean=25.6) and mild exacerbations (62.0) compared with their current health (81.0). The study data are, however, limited from a health economic perspective by the use of a VAS rating system which is generally considered less suitable for eliciting utility scores for use in economic evaluation. Despite that, the results are quite close to the results from the present study.

All three HRQL measures in this study indicate the very substantial burden associated with experiencing exacerbations. The analyses indicate that the MAQLQ and ASUI scores for exacerbation patients and hospitalised patients were significantly lower at both time points compared to the rest of the group. Those people who experienced an exacerbation during the study had significantly lower mAQLQ and ASUI scores at baseline compared to the others, but this did not get significantly worse during the course of the study. These patients' scores did not significantly decline between baseline and four weeks despite experiencing an exacerbation. In contrast the EQ$5 D_{\text {index }}$ scores showed a significant decline for those patients experiencing an exacerbation between baseline and four weeks. The MAQLQ and ASUI (which don't capture this additional decrement) may be exhibiting a floor effect for asthma patients who are this severe. Juniper and colleagues have reported (after the present study was initiated) that the AQLQ (and so by definition the $\mathrm{mAQLQ}$ ) is primarily designed to measure the day-to-day concerns of asthma patients and is not suitable for capturing the rapidly changing nature of an exacerbation..$^{15}$ To measure the impact of an exacerbation that requires admission to hospital, Juniper et al. have developed the Acute AQLQ. However, this measure is designed to be used in very acute settings (such as following admission to hospital) and so may not have worked well in the present study. In addition, there are a number of studies which report substantial variation in exacerbation rates but with no difference in AQLQ score. One study in particular reported a threefold variation in exacerbation rates but no measurable change on the AQLQ. ${ }^{16}$

The data have certain limitations and the study was limited by some missing data. It is difficult to determine the extent to which the deficit in HRQL in the exacerbation groups is due to the exacerbation or due to the fact that those patients who have an exacerbation have much worse asthma and so have worse HRQL. This study does provide some information regarding this issue. HRQL data were collected at baseline and four weeks later. Exacerbations during the four weeks were identified. Therefore, any difference between baseline and study-end HRQL is partly the result of the exacerbation and associated treatment. The patients who experienced exacerbations and hospitalisations had significantly worse HRQL at the start of the study compared to the other patients, which supports previous findings. ${ }^{8}$ However, there was also evidence from the EQ-5D that they experienced an additional significant decrement in HRQL during the four weeks, which could be 
attributed in part to the exacerbation. This additional decrement was not found on the MAQLQ and the ASUI.

Prospective data were only collected for one month. This was designed to allow us to record the impact of exacerbations as they occurred, without requiring study participants to continue completing forms for many months. A relatively large number of exacerbations were recorded which was sufficient for the purposes of statistical analysis (although the hospitalisation group was small). Therefore, we feel that the design was justified.

Exacerbations in these patients probably results from a combination of poor compliance, inadequate treatment, and a lack of provision of self management strategies. We have found that collecting these types of data is a significant challenge both methodologically and practically. Poorly compliant patients are perhaps one of the hardest groups of patients from whom to collect observational data. However, using an observational study to record the burden of exacerbations avoids the potential bias introduced by clinical trial protocols. We believe that these data are therefore suitable for any modelling studies that are designed to capture the benefits of treatments which reduce exacerbations.

\section{Acknowledgements}

The authors thank the principal investigators and researcher at each site who gathered the data: Professor Rob Angus, University Hospital Aintree, Liverpool; Professor Fan Chung, Royal Brompton Hospital, London; Professor Ann Millar, Southmead Hospital, Bristol; and researchers at the Department of General Practice and Primary Care, University of Aberdeen.

Funding: This study was supported by funding from Novartis AG. The views expressed are solely those of the authors. The authors had full control over the content and analyses of the study and the preparation of the manuscript.

\section{Conflict of interest}

There are no existing or potential conflicts of interest to declare.

\section{References}

1. McFadden ER. Acute severe asthma. Am J Respir Crit Care Med 2003:168:740-59.

2. GINA - Global Initiative for Asthma: A practical guide for public health officials and health care Professionals. National Heart Lung and Blood Institute, NHI and WHO; www.ginasthma.com. Date last updated: Oct 2004. Date last accessed: 21 Feb 2005.

3. Bateman ED, Boushey HA, Bousquet J, et al GOAL Investigators Group. Can guideline-defined asthma control be achieved: The gaining optimal asthma control study. Am J Respir Crit Care Med 2004:170:836-44.

4. Burr ML, Wat D, Evans C, Dunstan FD, Doull IJ; British Thoracic Society Research Committee. Asthma prevalence in 1973, 1988 and 2003. Thorax 2006;61(4):296-9.

5. Rabe KF, Vermeire PA, Soriano JB, Maier WC. Clinical management of asthma in 1999: the Asthma Insights and Reality in Europe (AIRE) study, Eur Respir J 2000:16(5):802-07.

6. Hayden ML, Chipps BE, Dolan CM, Johnson C for the TENOR Study Group. Asthma control in patients with severe or difficult-to-treat asthma. Proc Am Thoracic Soc 2002.

7. Slavin RG, Haselkorn T, Lee JH, Zheng B, Deniz Y, Wenzel S. Asthma in older adults: observations from the Epidemiology and Natural History of Asthma: Outcomes and Treatment Regimens (TENOR) study Ann Allergy Asthma Immun 2006;96:406-14.

8. Magid DJ, Houry D, Ellis J, Lyons E, Rumsfeld JS. Healthrelated quality of life predicts emergency department utilization for patients with asthma. Ann Emerg Med 2004; 43(5):551-7.

9. Scottish Intercollegiate Guidelines Network and British Thoracic Society, British Guideline on the Management of Asthma: Thorax 2003;58Suppl 1.

10. Juniper EF, Guyatt GH, Cox FM, Ferrie PJ, King DR. Development and validation of the mini asthma quality of life questionnaire. Eur Respir J 1999:14:32-8.

11. Brooks R. EuroQol: the current state of play. Health Pol 1996;37:53-72.

12. Kind $\mathrm{P}$, Brooks $\mathrm{R}$, Rabin $\mathrm{R}$ (Eds.) EQ-5D concepts and methods: a developmental history 2005 Springer, Dordrecht, Netherlands.

13. Revicki DA, Leidy NK, Brennan-Diemer F, Sorensen S, Togias A. Integrating patient preferences into health outcomes assessment: the multiattribute Asthma Symptom Utility Index. Chest 1998;114(4):99-1007.

14. Andersson $F$, Borg $S$, Ståhl $E$. The impact of exacerbations on the asthmatic patient's preference scores. J Asthma 2003;40(6):615-23.

15. Juniper EF Klas Svensson, Mörk AC, Ståhl E Measuring Health-Related Quality of Life in Adults During an Acute Asthma Exacerbation, Chest 2004;125:93-7.

16. Green $\mathrm{RH}$, Brightling $\mathrm{CE}$, McKenna $\mathrm{S}$, et al. Asthma exacerbations and sputum eosinophil counts: a randomised controlled trial. Lancet 2002;360:1715-21.

Available online at http://www.thepcrj.org 